\title{
X-RAY AND GAMMA-RAY SIGNATURES OF WOLF-RAYET SUPERNOVA EXPLOSIONS
}

\author{
LIH-SIN THE and DONALD D. CLAYTON \\ Department of Physics and Astronomy \\ Clemson University, Clemson, SC 29634, U.S.A. \\ ADAM BURROWS \\ Departments of Physics and Astronomy \\ University of Arizona, Tucson, AZ 85721, U.S.A.
}

\begin{abstract}
It is widely speculated that a Type Ib supernova is the explosion of a WolfRayet star. We calculate the X-ray and gamma-ray signatures of models of that type, assuming all hard photons to have originated with $\mathrm{Ni}$ decay chains, in hopes of providing diagnostics of the exposed-core models of massive stars, which constitute one model of the Wolf-Rayet stars, calculated by Ensman and Woosley (1988). These provide the characteristic luminosity peak and light curve of Type Ib supernovae for helium-core masses between 4 and $6 \mathrm{M}_{\odot}$. We compute gamma-ray line shapes and fluxes and the Comptonized X-ray continuum resulting from the decay of the radioactive ${ }^{56} \mathrm{Co}$ and ${ }^{57} \mathrm{Co}$ isotopes that are synthesized by the explosion of the presupernova star (the suggested Wolf-Rayet or postWolf-Rayet star) with a Monte Carlo transport code. The expansion velocity, the total mass of the ejecta, the radial mixing of radioactivity in that ejecta, and the ${ }^{56} \mathrm{Ni}$ yield effect both the strength and the evolution of the hard radiation. With the anticipated launch of Gamma Ray Observatory, we can hope to detect Type Ib supernovae to distances of 3 $\mathrm{Mpc}$ and utilize the characteristics of the gamma lines and X-ray spectrum to distinguish between differing Type Ib supernova models and to address their suggested relationship to Wolf-Rayet stars.
\end{abstract}

\section{Introduction}

Controversy surrounds interpretation of the nature of the Wolf-Rayet stars. Maeder and Meynet (1987) and Langer (1989) argue that cores of massive evolved stars, exposed by large mass loss during the Wolf-Rayet process, provide the characteristics and anomalous abundances of the observed WR stars. Underhill and her colleagues (Bhatia and Underhill 1986, 1988, 1989; Underhill, Gilroy, and Hill 1990) sternly challenge that interpretation, arguing that the abundances are not in fact anomalous and that several other features (e.g. surface brightness) are not in accord with such models. They prefer hydrogen-burning $B$ stars in binaries leading to the observed WR phenomenon. Paralleling this dispute is the 
emergence of a new class of supernovae, now called Type Ib because they were originally classed Type I owing to absence of hydrogen lines. Wheeler and Levreault (1985) discussed previous analysis of Type Ib structures and advanced the now common paradigm-that Type Ib undergo core collapse and bounce, like Type II, but are small dense objects having light curves dominated by ${ }^{56}$ Co decay, like Type I. Ensman and Woosley (1988) have constructed detailed evolutionary models of Type $\mathrm{Ib}$, finding that 4-to-6 $\mathrm{M}_{\odot}$ cores of evolved stars of initially greater mass may reproduce satisfactorily the Type Ib light curves, though subject to the same physics uncertainties that surround the actual Type II core bound mechanism. Extensive mass loss during the WR lifetime is supposed to reduce the final mass to 4-to-6 $\mathrm{M}_{\odot}$ in time for the final Type Ib explosion. We will call these progenitors Wolf-Rayet stars, remembering that the identification is contested.

Our contribution to this evolving debate is to explore the usage of gamma-ray lines to elucidate the true structural nature of the Type Ib phenomenon, and thereby its relationship to WR stars. Analysis of supernova structure using gamma-ray line and X-ray continuum has been done by many authors (Clayton, Colgate, and Fishman 1969; Colgate and McKee 1969; Clayton 1974; Ambwani and Sutherland 1988; Woosley and Pinto 1988; Chan and Lingenfelter 1988; Bussard, Burrows, and The 1989: Kumagai et al.1989). However, due to uncertainty of the Type Ib supernovae model, no work has been published predicting the expected gamma-ray line and X-ray fluxes of Type Ib supernovae. In this paper we do this by applying a Monte-Carlo transport code (The, Burrows, and Bussard 1990) to the Type Ib models calculated by Ensman and Woosley (1988). The sources of hard photons are the decay chains ${ }^{56} \mathrm{Ni} \rightarrow{ }^{56} \mathrm{Co} \rightarrow{ }^{56} \mathrm{Fe}$ and ${ }^{57} \mathrm{Ni} \rightarrow{ }^{57} \mathrm{Co} \rightarrow{ }^{57} \mathrm{Fe}$ (Clayton, Colgate, and Fishman 1969; Clayton 1974; The, Burrows, and Bussard 1990)

\section{Models}

Ensman and Woosley (1988; hereafter referred to as EW), find that He-cores of 4-to-6 $\mathrm{M}_{\odot}$ give the best fit to the available data of Type Ib peak magnitudes and light curves, although each of their models has its own problems in fitting the data. The Wolf-Rayet supernovae explosion models we use in this paper are those 4-to-6 $\mathrm{M}_{\odot}$ models of $\mathrm{EW}$ that produce the best light curves for Type Ib supernovae. Model 4A of EW (hereafter referred to as WR4A) which was initially a $15 \mathrm{M}_{\odot}$ main sequence star is assumed to have been reduced to a 4 $\mathrm{M}_{\odot}$ Wolf-Rayet star that finally ejects its last $2.68 \mathrm{M}_{\odot}$ of mass with an explosion energy of $0.5 \times 10^{51}$ ergs. This model produces $0.06 \mathrm{M}_{\odot}$ of ${ }^{56} \mathrm{Ni}$ and it is found to be somewhat too faint when compared with SN1983N peak magnitudes (EW). Model 6C (hereafter referred to as WR6C) was a $20 \mathrm{M}_{\odot}$ main sequence star reduced to a $6.2 \mathrm{M}_{\odot}$ Wolf-Rayet star that in its explosion of $2.7 \times 10^{51}$ ergs ejects $4.5 \mathrm{M}_{\odot}$ and produces $0.16 \mathrm{M}_{\odot}$ of ${ }^{56} \mathrm{Ni}$. It produced a good fit to SN1983N light curve (EW).

Because the SN1987A light curve was powered by radiactive decay of ${ }^{56} \mathrm{Co}$ (Catchpole et al.1988; Whitelock et al.1988) and because the early appearance of gamma-ray line showed that ${ }^{56}$ Co was mixed radially up to its hydrogen envelope (Sunyaev et al.1987; Matz et al.1988; Leising 1988; Sandie et al.1988; Cook et al.1988; Wilson et al.1988; Teegarden et 
al.1988; Nomoto et al.1988; Pinto and Woosley 1988; The, Burrows, and Bussard 1990), in our analysis we also evaluate mixed models; in model WR4Afm and WR6Cfm we mix all material in those entire stars uniformly ( fm meaning "fully mixed") and in model WR6Chm, the star inside the He burning shell is mixed uniformly. Such mixing enhances significantly the early X-ray continuum and gamma-ray line fluxes, but it now seems more realistic than an unmixed model, as demonstrated by SN1987A. It is also of interest to seek differences in the X-ray continuum and gamma-ray line fluxes between models having different speeds of expansion; for this we constructed model WR4Ab, which has all the characteristics of WR4A except its speed of expansion is $\sqrt{3}$ times the speed of the original model. We increase the speed of expansion of WR4A by this amount in order to make WR4A more like WR4B of EW, which provides the best overall fit to 1983N. We expect that the parameter space we explore (mixing and speed) covers most of the possibilities for this type of model of Type Ib supernovae. Many of the results we present here (flux ratios and energy fractions) do not depend on the amount of ${ }^{56} \mathrm{Ni}$ and, as $\mathrm{EW}$ conclude, this type of model ranges only from 4 to $6 \mathrm{M}_{\odot}$ helium-core stars, to which it is limited by the widths of bolometric light curves. We find that the characteristics of WR4Ab fall between those of WR4A and WR6C, since the expansion speed of WR4Ab is between that of these models, and also that the behavior of WR6Chm is between that of WR6C and WR6Cfm.

\section{Results}

The gamma-ray luminosities of these Type Ib supernovae are encouragingly bright. Figure 1 shows that just as for the optical luminosity, Type Ib supernovae 847-gamma-ray-line light curves falling between those of Type Ia and Type II supernovae. For Type Ia model, we show model W7 of Nomoto, Thielemann, and Yokoi (1984), where its gamma-ray and $\mathrm{X}$-ray continuum fluxes have been reported recently by Burrows and The (1990). The Type II model is the model W10hmm of Pinto and Woosley $(1988 \mathrm{a}, \mathrm{b})$ which is a successful model for almost the entire spectrum emitted by SN1987A. We also note that the peak time of gamma-ray-line light curves of Type Ib supernovae is later than for the Type Ia but earlier than that of the Type II. The $847 \mathrm{keV}$ maximum fluxes for this type of WolfRayet supernova explosion range from $\sim 3 \times 10^{-5}$ to $\sim 4 \times 10^{-4}$ photons $/ \mathrm{cm}^{2} s$ for explosions at our standard reference distance of $1 \mathrm{Mpc}$, which means with OSSE (Oriented Scintillation Spectrometer Experiment, Kurfess et al.1983) on Gamma Ray Observatory (with detection limit of $3 \times 10^{-5}$ photons $/ \mathrm{cm}^{2} \mathrm{~s}$ ) we can hope to detect extragalactic Type Ib supernovae gamma-ray lines to $\sim 3 \mathrm{Mpc}$.

In determining the gamma line profiles, we employ the analytic formalism developed by Bussard, Burrows, and The (1989). In Table 1 we show the fluxes at $1 \mathrm{Mpc}$ and properties of the $847 \mathrm{keV}$ gamma line at the time of maximum for those models shown in Figure 1. We find also that the FWHM (full width at half maximum) of gamma line profiles for Wolf-Rayet supernova explosions is between the FWHM's of Type Ia and Type II. One sees from Table 1 that for all supernova types the products $\mathrm{T}_{\max } \times \mathrm{FWHM}$ are approximately equal, which is because $\mathrm{T}_{\max } \sim 1 / v$ and FWHM $\propto v$. FWHM of the Ib models studied lie 


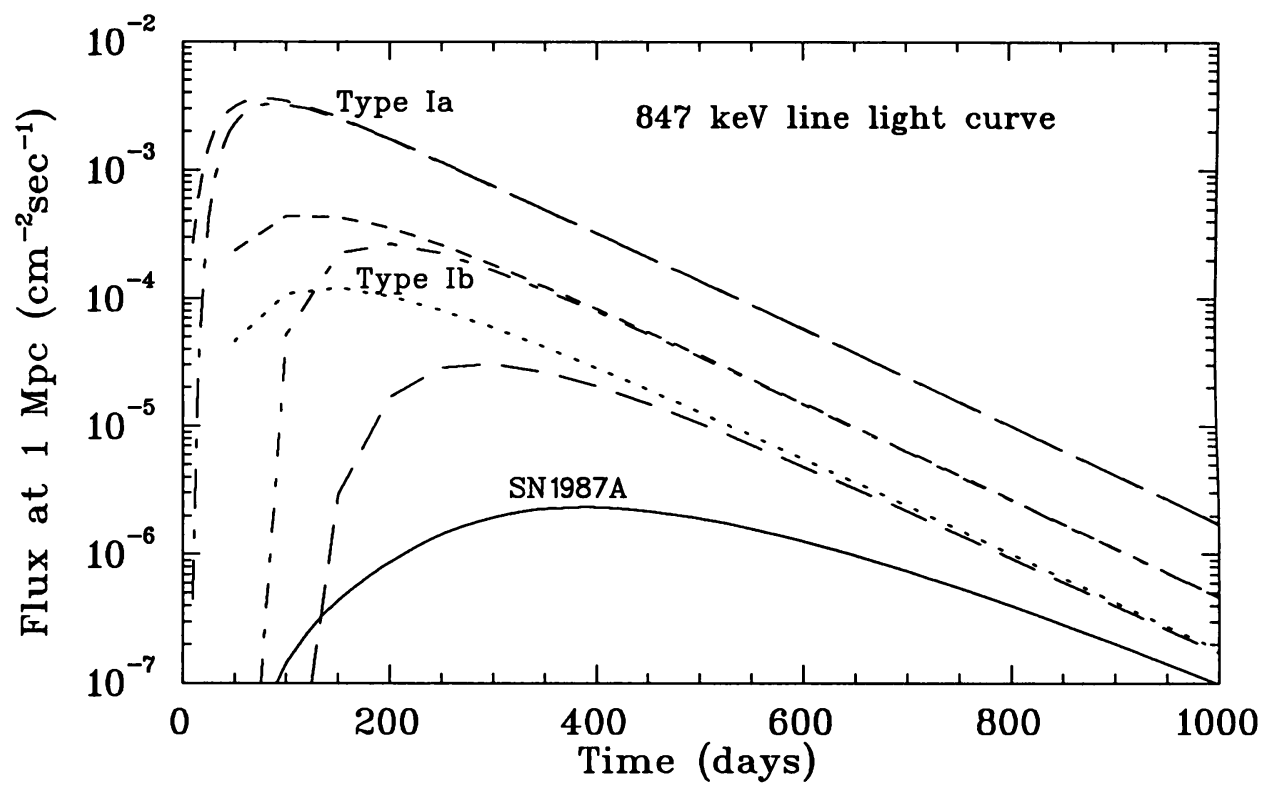

Figure 1. The $847 \mathrm{keV}$ Gamma line light curves of different types of supernovae at distance of 1 Mpc: (Ia) W7; (Ib) WR6C upper and WR4A lower; (SN1987A for Type II) W10hmm. Brighter branches of W7, WR6C, and WR4A are fully mixed counterparts of those models.

between 6 to $23 \mathrm{keV}$ and their shapes depend on the ${ }^{56} \mathrm{Ni}$ distribution. For a photon source distributed in a thin shell, the gamma line profile at maximum has a box shape, while a uniformly distributed source has a parabolic profile (Burrows and The 1990).

Table 1. The $847 \mathrm{keV}$ gamma-ray line maximum fluxes at $1 \mathrm{Mpc}$, the time at peak $\left(\mathrm{T}_{\max }\right)$, FWHM at $\mathrm{T}_{\max }$, and the spectral shape for different types of supernovae.

\begin{tabular}{|c|l|c|c|c|l|}
\hline Type & Model & $\mathrm{T}_{\max }$ (days) & $\mathrm{F}_{\max }^{1 \mathrm{Mpc}\left(\mathrm{cm}^{-2} \mathrm{~s}^{-1}\right)}$ & FWHM(keV) & shape \\
\hline Ia & W7 & 90 & $3.3 \times 10^{-3}$ & $\sim 26.9$ & parabolic \\
& W7 mixed & 80 & $3.6 \times 10^{-3}$ & $\sim 30.0$ & parabolic \\
Ib & WR4A & 275 & $3.1 \times 10^{-5}$ & $\sim 5.9$ & box \\
& WR4A mixed & 120 & $1.2 \times 10^{-4}$ & $\sim 13.9$ & parabolic \\
& WR6C & 180 & $2.8 \times 10^{-4}$ & $\sim 17.4$ & box \\
& WR6C mixed & 100 & $4.4 \times 10^{-4}$ & $\sim 23.2$ & parabolic \\
II & W10hmm & 390 & $2.3 \times 10^{-6}$ & $\sim 5.8$ & parabolic \\
\hline
\end{tabular}

In Figure 2 we show our calculations of the photon spectrum for four models (WR4A, WR6C, and their fully mixed counterparts) at two different times (100d and 200d). Continuum fluxes greater than $2 \times 10^{-7} \mathrm{~cm}^{-2} \mathrm{~s}^{-1} \mathrm{keV}^{-1}$ above $\mathrm{E}=50 \mathrm{keV}$ have good hopes for detection by OSSE. One sees that the different continua at $100 \mathrm{~d}$ partially converge at $200 \mathrm{~d}$. Line fluxes and ratios between selected X-ray bands are in Table 2. 

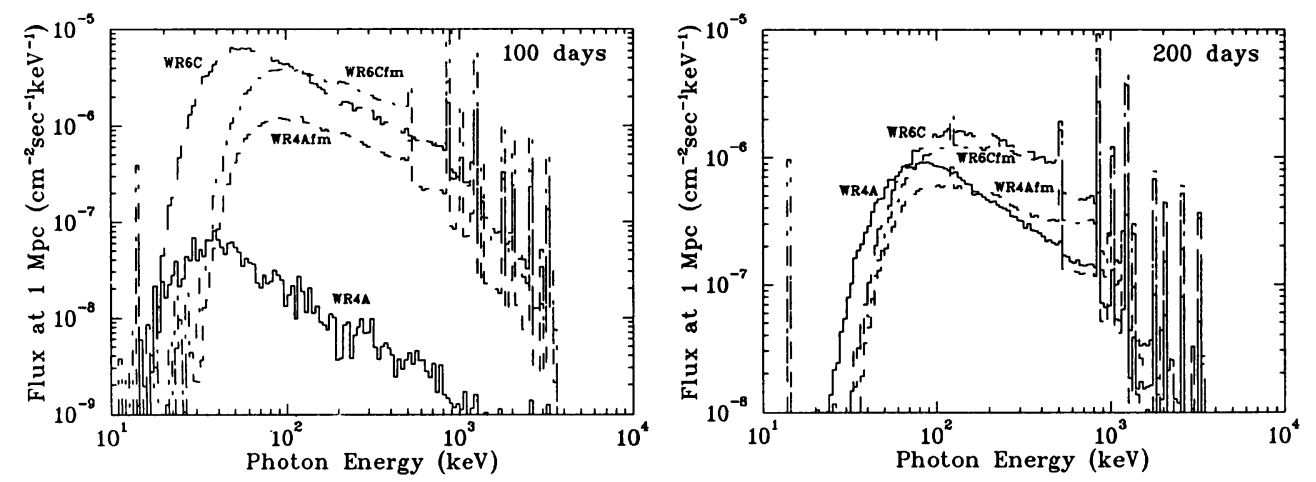

Figure 2. Continuous spectra for model WR4A, WR4Afm, WR6C, and WR6cfm at days 100 and 200. The heights of line-containing bins are plotted as the number of line photons (see Table 2) divided by the width of the energy bin into which they fall. The emergent photons are binned logarithmically from $1 \mathrm{keV}$ to $5 \mathrm{MeV}$ in 188 energy bins.

Table 2. Line fluxes $\left(10^{-5} \mathrm{~cm}^{-2} \mathrm{~s}^{-1}\right)$ for Models at $1 \mathrm{Mpc}$, the X-ray band ratios, and the ratio of the total X-ray and gamma-ray luminosities.

\begin{tabular}{|l|c|c|c|c|c|c|c|c|c|c|}
\hline Model & \multicolumn{2}{|c|}{$\mathrm{F}_{847}$} & \multicolumn{2}{c|}{$\mathrm{F}_{1238}$} & \multicolumn{2}{c|}{$\frac{F(50-100)}{F(550-700)}$} & \multicolumn{2}{c|}{$\frac{F(50-100)}{F(350-400)}$} & \multicolumn{2}{c|}{$f_{X} / f_{\gamma}$} \\
\hline & $100 \mathrm{~d}$ & $200 \mathrm{~d}$ & $100 \mathrm{~d}$ & $200 \mathrm{~d}$ & $100 \mathrm{~d}$ & $200 \mathrm{~d}$ & $100 \mathrm{~d}$ & $200 \mathrm{~d}$ & $100 \mathrm{~d}$ & $200 \mathrm{~d}$ \\
\hline WR4A & $4 \times 10^{-4}$ & 1.69 & 0.002 & 1.79 & 3.0 & 1.8 & 5.9 & 3.2 & 3.7 & 1.1 \\
WR4Afm & 10.9 & 10.4 & 8.32 & 7.65 & 1.5 & 1.3 & 2.0 & 1.4 & 0.43 & 0.28 \\
WR4Ab & 0.803 & 9.04 & 1.11 & 7.13 & 2.8 & 0.68 & 4.9 & 0.95 & 1.7 & 0.42 \\
WR6C & 5.17 & 26.7 & 6.03 & 20.5 & 2.7 & 0.73 & 5.1 & 1.0 & 1.4 & 0.34 \\
WR6Chm & 10.7 & 25.8 & 9.84 & 19.8 & 2.3 & 0.73 & 3.6 & 0.96 & 0.50 & 0.36 \\
WR6Cfm & 43.8 & 35.4 & 32.8 & 25.6 & 1.5 & 0.85 & 1.8 & 0.82 & 0.36 & 0.21 \\
\hline
\end{tabular}

The ratio of the total X-ray and gamma-ray luminosities, $f_{X} / f_{\gamma}$, is also significant because it reveals the time during the early light curve when the X-ray flux is dominant and more easily detected. That same ratio also reveals the extent of mixing (e.g. Leising 1988). We find that $f_{X} / f_{\gamma}$ for fully mixed models is monotonically decreasing after 50 days and $f_{X} / f_{\gamma} \leq 0.5$. The WR4Afm example in Figure $3 \mathrm{a}$ is such a model. Figure 3a also shows several fractions of total radioactive power going into differing channels as a function of time. For an unmixed model, on the other hand, $f_{X} / f_{\gamma}$ is $>0.5$ for times between 50 and 150 days. For any prescription of mixing and kinetic energy, at times after the peak $\left(\mathrm{T}_{\text {max }}\right)$ of the $847 \mathrm{keV}$ line light curve, $f_{X} / f_{\gamma}<0.5$. The effect of mixing dramatically reduces the value of $f_{X} / f_{\gamma}$ for times between 50 and 200 days and this dependence is more pronounced than is that on the speed of expansion. The effect of mixing and expansion on $f_{\text {opt }}\left(=1-f_{X}-f_{\gamma}\right)$ is similar to that on $f_{X} / f_{\gamma}$.

We show in Figure $3 \mathrm{~b}$ the flux ratios for selected continuum bands as a function of time for fully mixed model WR4Afm. Table 2 shows the effect of mixing and the increased speed of expansion at both $100 \mathrm{~d}$ and $200 \mathrm{~d}$ on the $F_{50-100} / F_{550-700}$ and $F_{50-100} / F_{350-400} \mathrm{X}$-ray- 

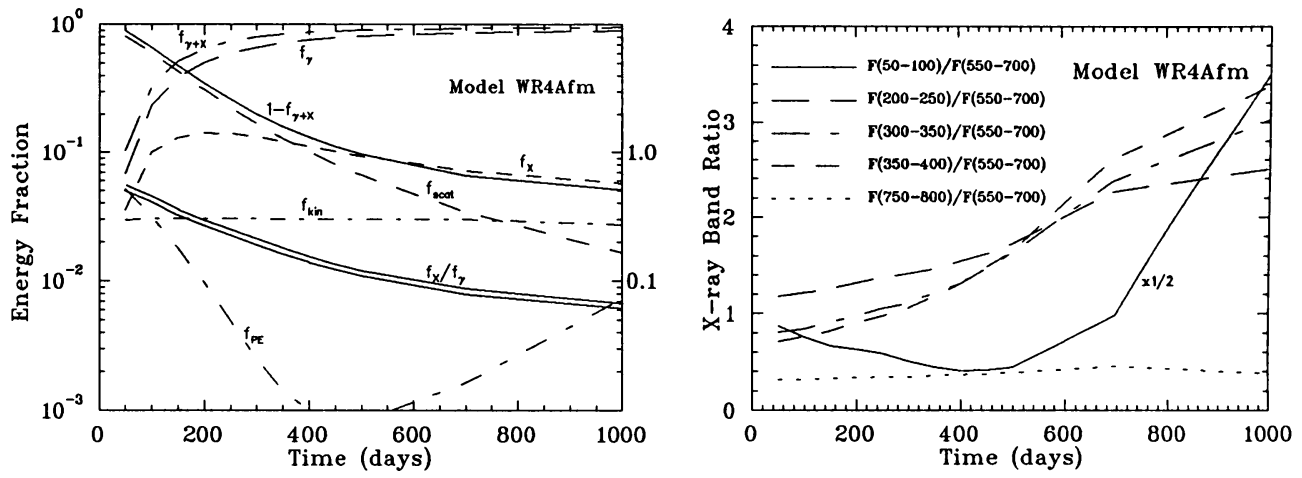

Figure $3 a$. Fractions of the total radioactive power that emerge as $X$-ray $\left(f_{X}\right)$ and gamma-lines $\left(f_{\gamma}\right)$, their total $\left(f_{\gamma+X}\right)$, and the fractions deposited in the plasma by scattering $\left(f_{\text {scat }}\right)$, photoelectric absorption $\left(f_{P E}\right)$, and the ${ }^{56}$ Co positron $\left(f_{k i n}\right)$ as functions of time for model WR4Afm. The double curve shows the ratio of $f_{x} / f_{\gamma}$ on the right ordinate. Figure $3 b . X$-ray band ratios.

band ratios. These band ratios reflect the slope of the X-ray spectrum in Figure 2. Mixing and increased speed cause smaller values of $F_{50-100} / F_{550-700}$ for times when ${ }^{57} \mathrm{Co}$ is not a major contributing source to this band. For a fully mixed model, $F_{50-100} / F_{550-700}$ is $<2$ and decreases after 50 days, unless ${ }^{57} \mathrm{Co}$ is present. Its increase in Figure $3 \mathrm{~b}$ after day 400 is mostly due to the contribution from the 122 and $136 \mathrm{keV}{ }^{57} \mathrm{Co}$ gamma lines, which are beginning to assume a greater role. The other band flux ratios increase with time for any of these models until that time when most of the photons escape directly (only WR6Cfm of our models starts to decrease at 700 days). The crossover of X-ray-band ratios shown in Figure $3 \mathrm{~b}$ near $550 \mathrm{~d}$ is due to the increasing contribution of three-photon positronium decays at later times. At early times, most of the photons in the bands below $500 \mathrm{keV}$ come from scattering of the 847 and $1238 \mathrm{keV}{ }^{56} \mathrm{Co}$ gamma lines and from direct escape and scatterings of three photon positronium decays. But at late time most of 847 and 1238 $\mathrm{keV}$ gamma lines escape directly, so that their contributions to the X-ray bands become much smaller than direct escape photons from positronium decays.

The X-ray-band ratios and the ratio of X-ray and gamma-ray luminosites of WR4Ab and WR6C are very similar, suggesting that the evolution of these ratios can identify the speed of expansion of the supernova. $f_{X} / f_{\gamma}$ for a higher expansion speed model can be roughly scaled from a lower expansion model by contracting the time scale in proportion to $1 / v$. At early time, the X-ray-band ratios for energies larger than $200 \mathrm{keV}$ are similar for mixed and unmixed models and also for slow and fast expansion models, but these ratios become more distinguishable at later times. Differentiating between models is easier in the low $50-100 \mathrm{keV}$ band since its intensity strongly depends on the distribution of elements having high photoelectric cross sections.

We have demonstrated not only that the fluxes, peak time, and FWHM can be used to distinguish different types of supernovae, but also that gamma-line fluxes, shapes, FWHM, $\mathrm{X}$-ray-band ratios, ratio of X-ray and gamma-ray luminosities, and the fraction of energy 
deposited into the ejecta can reveal the extent of mixing in the ejecta and its speed of expansion. Other characteristics that can be utilized for X-ray diagnosis of a supernovae are line ratios, the X-ray cutoffs, the energy of the peak continuum flux, and turn-on times (Burrows and The 1990). A more complete account of these details is under preparation by us. We can offer hope that good measurements of a Type Ib supernova will reveal its structure, so that if its progenitor was indeed a Wolf-Rayet star, the state of its evolution and prior mass loss may be circumscribed by these measurements.

Acknowledgments: L-ST and DDC research was supported by Naval Research Laboratory Grant N00014-89-J-2034 under the NASA Contract for the OSSE Spectrometer DPRS-10987C on GRO. AB research was supported by the National Science Foundation through grant no. AST-89-14346 and by NASA through the Long Range Space Astrophysics Research Program. We are grateful to Lisa Ensman for providing us with model 4A, 6C, and 8A. L-ST would like to thank TRIUMF, especially C.J. Kost for providing TRIUMF graphics software that we use in preparing the graphs in this paper.

\section{References}

Arnett, D.W. (1979) 'On the theory of Type I supernovae', Astrophys. J., L37-L40.

Ambwani, K. and Sutherland, P. (1988) 'Gamma-ray spectra and energy deposition for Type Ia supernovae', Astrophys. J., 325, 820-827.

Bhatia, A.K. and Underhill, A.B. (1986) 'The statistical equilibrium of hydrogen and helium in a radiation fiels, with an application to interpreting Wolf-Rayet spectra', Astrophys. J. Suppl., 60, 323-356.

Bhatia, A.K. and Underhill, A.B. (1988) 'Carbon and nitrogen lines in the spectra of Wolf-Rayet stars', Astrophys. J. Suppl., 67, 187-223.

Bussard, R., Burrows, A., and The, L.-S.' (1989) 'SN1987A gamma-ray line profiles and fluxes', Astrophys. J., 341, 401-413.

Burrows, A. and The, L.-S. (1990) 'X-ray and gamma-ray signatures of Type Ia supernovae', to appear in Astrophys. $J$.

Catchpole, R.M., Whitelock, P.A., Feast, M.W., Menzies, J.W., Glass, I.S., Marang, F., Laing, J.D., Spencer Jones, J.H., Roberts, G., Balona, L.A., Carter, B.S., Laney, C.D., Lloyd Evans, T., Sekiguchi, K., Hutchinson, M.G., Maddison, R., Albinson, J., Evans, A., Allen; D.A., Winkler, H., Fairall, A., Corbally, C., Davies, J.K., and Parker, Q. (1988) 'Spectroscopic and photometric observations of SN1987a -II. days 51 to 134', Monthly Notices Roy. Astron. Soc. 231, 75p-89p.

Chan, K.W. and Lingenfelter, R.E. (1988) 'Gamma ray lines from supernovae', in N. Gehrels and G. Share (eds.), Nuclear Spectroscopy of Astrophysical Sources, AIP Conf. Proc. 170, New York, pp. 110-115.

Clayton, D.D. (1974) 'Line ${ }^{57}$ Co gamma rays: New diagnostic of supernova structure', Astrophys. J., 188, 155-157.

Clayton, D.D., Colgate, S.A., and Fishman, G. (1969) 'Gamma ray lines from young supernova remnants', Astrophys. J., 155, 75-82.

Colgate, S.A. and McKee, C. (1969) 'Early supernova Luminosity', Astrophys. J., 157, 623-643. 
Cook, W.R., Palmer, D.M., Prince, T.A., Schindler, S., Starr, C.H., and Stone, E.C. (1988) 'An imaging observation of SN 1987A at gamma-ray energies', Astrophys. J. Letters, 334, L87-L90.

Ensman, L.M. and Woosley, S.E. (1988) 'Explosions in Wolf-Rayet stars and Type Ib supernovae', Astrophys. J., 333, 754-776.

Kumagai, S., Shigeyama, T., Nomoto, K., Itoh, M., Nishimura, J., Tsuruta, S. (1989) 'Gamma-ray, X-ray, and optical light from the cobalt and the neutron stars in SN 1987 A', Astrophys. J., 345, 412-422.

Kurfess, J.D., Johnson, W.N., Kinzer, R.L., Share, G.H., Strickman, M.S., Ulmer, M.P., Clayton, D.D., Dyer, C.S. (1983) 'The Oriented scintillation spectrometer experiment for the gamma-ray observatory', Adv. Space Res., 3, 109-112.

Langer, N. (1989) 'Standard models of Wolf-Rayet stars', Astron. Astrophys., 210, 93113.

Leising, M.D. (1988) 'Gamma-rays and X-rays from SN1987A', Nature, 332, 516-518.

Maeder, A. and Meynet, G. (1987) 'Grids of evolutionary models of massive stars with mass loss and overshooting. Properties of Wolf-Rayet stars sensitive to overshooting', Astron. Astrophys. 182, 243-263.

Matz, S.M., Share, G.H., Leising, M.D., Chupp, E.L., Vestrand, W.T., Purcell, W.R., Strickman, M.S., and Reppin, C. (1988) 'Gamma-ray line emission from SN1987A', Nature, 331, 416-418.

Nomoto, K., Thielemann, F.-K., and Yokoi, K. (1984) 'Accreting white dwarf models for Type I supernovae. III. carbon deflagration supernovae', Astrophys. J., 286, 644-658.

Pinto, P.A. and Woosley, S.E. (1988) 'X-ray and gamma-ray emission from supernova 1987A', Astrophys. J., 329, 820-830.

Sandie, W.G., Nakano, G.H., Chase, Jr, L.F., Fishman, G.J., Meegan, C.A., Wilson, R.B., Paciesas, W.S., and Lasche, G.P. (1988) 'High-resolution observations of gamma-ray line emission from SN 1987A', Astrophys. J. Letters, 334, L91-L94.

Sunyaev, R., Kaniovsky A., Efremov, V., Gilfanov, M., Churazov, E., Grebenev, S., Luznetsov, A., Melioransky, A., Yamburenko, N., Yunin, S., Stepanov, D., Chulkov, I., Pappe, N., Boyarskiy, M., Gavrilova, E., Loznikov, V., Prudkoglyad, A., Rodin, V., Reppin, C., Pietsch, W., Engelhauser, J., Trümper, J., Voges, W., Kendziorra, E., Bezler, M., Staubert, R., Brinkman, A.C., Heise, J., Meis, W.A., Jager, R., Skinner, G.K., Al-Eman, O., Patterson, T.G., Wilmore, A.P. (1988) 'Discovery of hard X-ray emission from supernova 1987 A', Nature, 330, 227-229.

Teegarden, B.J., Barthelmy, S.D., Gehrels, N., Tueller, J., Leventhal, M., and MacCallum, C.J. (1989) 'Resolution of the 1,238 keV gamma-ray line from supernova 1987A', Nature, 339, 122-123.

The, L.-S., Burrows, A., and Bussard, R. (1990) 'X-ray and gamma-ray fluxes from SN1987A', Astrophys. J., 352, 731-740.

Underhill, A.B., Gilroy, K.K., and Hill, G.M. (1990) 'About the eclipsing Wolf-Rayet binary HD214419', Astrophys. J., 351, 651-665.

Wheeler, J.C. and Levreault, R. (1985) 'The peculiar type I supernova in NGC991', Astrophys. J. (Letters), 294, L17-L24.

Woosley, S.E. and Pinto, P.A. (1988) 'Gamma-producing radioactivities from supernovae', in N. Gehrels and G.H. Share (eds.), Nuclear Spectroscopy of Astrophysical Sources, AIP Conf. Proc. 170, New York, p98-109. 


\section{DISCUSSION}

Filippenko: Can you distinguish between a 3-4 $M_{\odot}$ helium core with no clumping and mixing, and a $6 M_{\odot}$ helium core with clumping and mixing?

The: Here we compare model WR4A and WR6Cfm with clumping. Modeling clumps such as done in the paper of Nomoto et al. (1989) for SN1987A (where clumping happens inside the $\mathrm{He}$-core), at the early time when the ejecta is still thick, most of X-ray and $\gamma$-ray radiation come from sources near the surface of ejecta. Therefore, at early time the value of X-ray band ratio and $f x / f \gamma$ for WR6Cfm with clumping do not differ much from WR6Cfm. At later time when sources from deeper layer dominate, the value of X-ray band ratio and $f x / f \gamma$ increases to a larger value than in WR6Cf. $f x / f \gamma$ in WR6Cfm with clumping is larger than $f x / f \gamma$ in WR6Cfm, but cannot be larger than the value in WR4A. However, since clumping effects add significantly to low energy X-rays, the X-ray band ratio $F(50-100) / F(550-700)$ can increase as high as in WR4A.

Montmerle: Have you considered the X-ray emission from longer-lived isotopes like ${ }^{44} \mathrm{Ti}$ ? Or do they appear too late to be of importance, given the sensitivity limit of GRO?

The: In this calculation we do not include other radioactive sources, only ${ }^{56} \mathrm{Ni}$ and ${ }^{57} \mathrm{Ni}$ decay chains are considered. Other sources are less abundant. It is still important to detect and to know the amount of these radioactive sources in order to have a better understanding of nucleosynthesis theory.

Nomoto: If you take into account clumpiness to the extent as made by Ensman and Woosley, how large is its effect on your results?

The: Clumping increases the X-ray and $\gamma$-ray total luminosities. This effect increases significantly the low-energy X-ray, i.e., $F(50-100) / F(550-700)$, but $f x / f \gamma$ increases slightly.

Langer: I would like to point out that a $\mathrm{He}$-core of (e.g.) $5 M_{\odot}$, which originates from a relatively low mass star $\left(\right.$ e.g. $\left.20 M_{\odot}\right)$, has a much different internal structure compared to a $5 M_{\odot}$ core which originates from a much more massive star (say, $60 M_{\odot}$ ). For this reason, your models which are based on the Ensman and Woosley calculations, might correspond to the binary scenario discussed by Nomoto rather than to the WR scenario for Type Ib supernova progenitors of Langer and Woosley ( $c f$. poster abstract)

The: Chiosi and Maeder have calculated that evolution of the material inside $\mathrm{He}$-burning does not depend on the hydrogen envelope. With and without removing the hydrogen, the results should be the same.

Shara: (1) Can GRO detect supernovae anywhere in the Milky Way? (2) What would we have learned about SN1987A had GRO been aloft there?

The: (1) With GRO sensitivity, it will be able to detect all type of supernovae in the Milky Way, unless the explosion happens at the other side of Galactic Center or is blocked by another object along the line of sight. (2) If we had GRO on orbit since the first day of SN1987A, we would have got an accurate measurement of $\gamma$-line light curve which can be used to uniquely determine the ${ }^{56} \mathrm{Ni}$ distribution and its density structure. Since GRO has a narrow FWHM detection limit, measurements of $\gamma$-line shapes can map the velocity distribution of radioactive sources. Clumping and mixing can also be determined by X-ray measurements. 


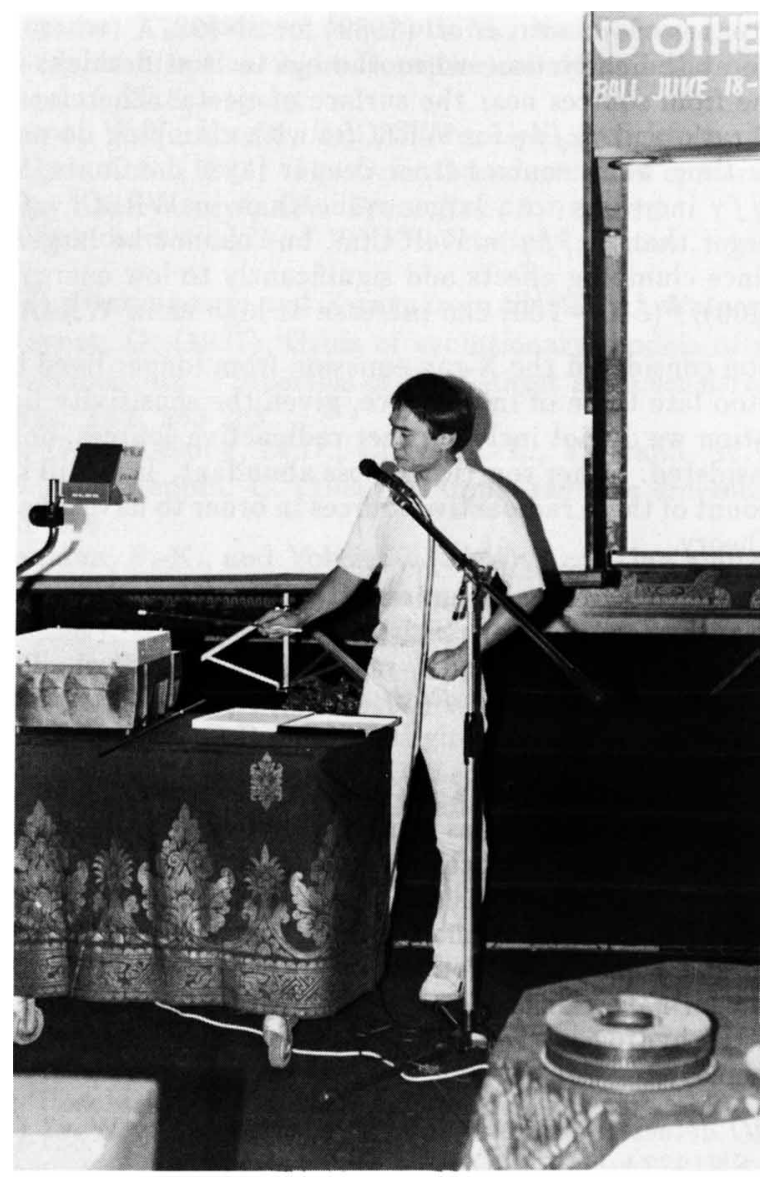

Lih-Sin The 